\title{
The education and training needs of advanced clinical practitioners: An exploratory, qualitative study
}

\author{
Suzanne Bench*1, Edward Baker ${ }^{2}$, Nicola Dover ${ }^{2,3}$, Brendan Garry², Winifred Nwosu², Shelley Peacock ${ }^{2}$, Natasha \\ Trenchard-Turner ${ }^{3}$, Sue Whaley², Timothy Worth², Geraldine A. Lee ${ }^{2}$ \\ ${ }^{1}$ School of Health and Social Care, London South Bank University, England and the Royal National Orthopaedic Hospital, \\ London, England \\ ${ }^{2}$ Florence Nightingale Faculty of Nursing, Midwifery \& Palliative Care, King's College, London, England \\ ${ }^{3}$ Departments of Emergency/Critical Care, King's College Hospital NHS Foundation Trust, London, England
}

Received: February 28, 2018

DOI: $10.5430 /$ jnep.v8n8p66
Accepted: March 23, 2018

Online Published: March 27, 2018

\begin{abstract}
Advanced clinical practitioners increasingly provide patient care in a variety of settings across the world. This paper reports a qualitative study exploring the training and education needs of these healthcare professionals in England. Four focus group discussions and one individual interview were conducted with a total sample of 17 people. Participants were adults enrolled on an Advanced Practice Masters programme at one Higher Education Institution or advanced clinical practitioners from across two large London National Health Service hospitals. Data collection took place March-April 2017. Following transcription, audio-recorded data were imported into Nvivo11 and subjected to a standard process of inductive thematic analysis. Three key themes were identified: Recognising advanced practice; Education for Advanced Practice; Programme delivery. Findings highlight the huge variation in titles, practice responsibilities and management structure, which make the development of a generic education programme challenging and the importance of flexibility key to success. At a time when public services are experiencing significant financial constraints, the need for improved collaborative practices, shared resources and a practice focus is considered vital for educating future advanced clinical practitioners worldwide.
\end{abstract}

Key Words: Education, Qualitative research, Advanced clinical practice nursing, Curriculum

\section{INTRODUCTION}

Internationally, many hospitals employ healthcare professionals who provide Advanced Practice (AP) to patients in a variety of settings. ${ }^{[1]}$ Increasing healthcare demands globally has led to the development of innovative attitudes toward the mix of skills in teams. Advanced Clinical Practitioners (ACPs) are experienced professionals from a range of healthcare backgrounds including nursing, pharmacy, paramedical science, physiotherapy and occupational therapy. ${ }^{[1,2]}$
These individuals can assess, diagnose and treat patients as autonomous practitioners in roles that were traditionally physician-led (for example, pharmacist-led heart failure clinics and musculoskeletal physiotherapists in emergency departments). ${ }^{[1,3,4]}$

Despite different international definitions, there is consensus that ACPs should be educated to Masters Level and have the requisite knowledge and skill related to clinical practice; management and leadership; education and research; and

\footnotetext{
*Correspondence: Suzanne Bench; Email: benchs@1sbu.ac.uk; Address: School of Health and Social Care, London South Bank University, 103 Borough Road, London, England.
} 
clinical competence. ${ }^{[1,2]}$ The continuously extending scope and breadth of the ACP role means that practitioners have unique training and education needs, which may not be met by traditional academic programmes. ${ }^{[5]}$ In addition, role expansion has left many current staff needing to extend their skills and knowledge. ${ }^{[2,3,6]}$ However, despite a plethora of literature on AP and a National multidisciplinary framework published in England, ${ }^{[2]}$ a scoping review undertaken by the authors identified only two previous small scale primary research studies, one conducted in Sweden ${ }^{[7]}$ and one in Finland ${ }^{[8]}$ specifically addressing the educational preparation of ACPs.

Within the United Kingdom (UK), the majority of healthcare professionals graduate following completion of a Bachelor's degree. A proportion of these people subsequently complete a Master's programme of study on a part-time basis (3-5 years), whilst also working full time in a clinical role. In addition, it is common for people to study ad-hoc modules of relevance to their own area of speciality without completing a full programme. Whilst a postgraduate "Advanced Practice" programme has existed for some years at the local Higher Education Institution (HEI), as part of a wider curriculum review project, it was necessary to ensure future curricula meet the needs of contemporary practitioners. Thus, like that of Bergström and Lindh ${ }^{[7]}$ the present study was framed by its regional context, with an aim to explore local ACPs' views and perceptions regarding:

- Current and future training and education needs

- The barriers and facilitators related to ACP training and education

\section{METHODS}

A qualitative methodology was employed with focus group (FG) discussions used as the predominant method of data collection, supplemented by individual interviews.

\subsection{Sample}

A total sample of 17 people took part in the study, 12 of whom were past or present students on the local AP programme (see Table 1).

Table 1. Participants

\begin{tabular}{llll}
\hline Focus group/Interview & No. participants & Gender & Profession \\
\hline Interview 1 (Int1) & 1 & Female $n=1$ & Paediatric nurse $\times 1$ \\
Focus Group 2 (FG2) & 5 & Female $n=3$, Male $n=2$ & Pharmacist $\times 1$, Adult nurses $\times 3$, Midwife $\times 1$ \\
Focus Group 3 (FG3) & 5 & Female $n=3$, Male $n=2$ & $\begin{array}{l}\text { Adult nurses } \times 2 \text {, Allied healthcare professionals } \\
\text { (AHPs) } \times 3 \text { (radiologist, healthcare scientists) }\end{array}$ \\
Focus Group 4 (FG4) & 3 & Female $n=2$, Male $n=1$ & Paediatric nurse $\times 1$, Adult nurse $\times 1$, Paramedic $\times 1$ \\
Focus Group 5 (FG5) & 3 & Female $n=2$, Male $n=1$ & Adult nurses $\times 2$, Paramedic $\times 1$ \\
\hline
\end{tabular}

\subsection{Inclusion and exclusion criteria}

\subsubsection{Inclusion criteria}

- Students enrolled on an Advanced Practice programme at the local Higher Education Institution.

- Advanced Clinical Practitioners or those directly responsible for them.

\subsubsection{Exclusion criteria}

- Medical or dentistry staff

To recruit participants, an email was sent to all those enrolled on an AP Masters programme at one Central London HEI. Information sessions were used to introduce the study to all those enrolling on modules within the programme. In addition, emails were sent to all past students and to ACP contacts from two large London National Health Service (NHS) Trusts. All emails included study information, an invite to participate and contact details for the research team to discuss potential involvement. A snowballing approach (ask- ing those recruited to identify other potential participants) was used as an additional recruitment strategy.

\subsection{Data collection methods and tools}

Data collection took place March-April 2017. Following best practice guidance ${ }^{[9,10]}$ four FG discussions and one semistructured individual interview were conducted by two members of the research team. One researcher (SB) moderated all discussions, whilst a second acted as a non-participant observer and note-taker. The audio-recorded discussions with student groups took place on university premises whereas those with ACP staff were held at a hospital education centre. A topic guide, which included a set of open-ended questions, was used to encourage participants to reflect on their experiences, share their views and critically explore the subject of interest. There were no interruptions during the process of data collection and all discussions were completed within the agreed one hour timeframe. Notes taken by the nonparticipant observer confirmed that everyone contributed 
well to the discussion and that there were no obvious power hierarchies, which impacted on group dynamics. Furthermore, although some student participants had attended during their lunchtime period, there was no evidence that this had affected their concentration or degree of participation.

\section{Topic guide}

\section{- INTRODUCTION}

- Check participants have read information sheet, signed consent form and understand process.

- Introductions using first names and role.

- Ground rules including confidentiality.

- General open questions will be followed by prompts as necessary.

- SECTION 1: Opening

- Encourage each participant to share their experiences of advanced practice, to discuss their roles and activities and the training and education they have received.

- SECTION 2: Focusing

- Encourage group to focus specifically on training and education: its content, its merits and limitations.

- Encourage group to discuss the way training/education was delivered, by whom, where, in what format and associated merits and limitations.

- SECTION 3: Forward planning

- Encourage group to consider needs of future practitioners; what additional training/education needs to be provided; how should it be provided?

- Encourage discussion of resources constraints and how these might affect delivery and be addressed.

- Ask for creative or innovative ideas that participants have or have previously experienced.

- Conclusion

(1) Is there anything else you would like to say/tell me about the topics we have covered?

(2) Is there anything you would like to ask me?

- Thank people for their time and contribution.

- Outline process of data analysis and dissemination.

\subsection{Data analysis}

Descriptive statistics (frequencies and percentages) were used to describe the sample. Interim analysis at the end of each FG or interview highlighted emerging themes and the degree of data saturation. Following professional transcription, 132 pages of transcribed audio-recorded data were amalgamated with six pages of written notes, imported into NVivo11 and subjected to a standard process of inductive thematic analysis. ${ }^{[11]}$ First level coding was undertaken by one person (TW) and second level coding and theme development by a second researcher (SB). Final categories and themes were reviewed by all members of the project team and a consensus approach used to resolve any differences in opinion. Selected anonymised quotes, highlighting key issues of significance, are reported as part of the results.

\subsection{Ethical considerations and funding}

Ethical approval was granted by the Psychiatry, Nursing \& Midwifery Research Ethics Panel within the local HEI (LRS-16/17-4259). The project was funded by the Faculty of Nursing, Midwifery \& Palliative Care, which hosts the AP programme. The project team included both academics and clinicians. The Focus Group (FG) moderator had no direct teaching responsibilities or contact with the student group. Pressure to participate was further avoided by use of the programme administration team for all direct communications.

Written informed consent was obtained prior to all FG discussions and interviews. As an incentive to take part and to compensate for their time, participants were entered into a draw to win a $£ 50$ shopping voucher and light refreshments were offered during discussions. Participants were informed that they did not have to answer any questions that made them uncomfortable and that they were free to leave, stop the interview or take a break at any point. Furthermore, although it was not possible to guarantee confidentiality by other group members, FG participants were asked not to share with non-participants anything discussed within the session.

\section{Findings}

Represented professions included those from nursing, midwifery, paramedical science, pharmacy, radiology and medical physics. Participants worked in acute hospital and community settings with adult and/or paediatric population groups (see Table 1). Some worked as part of a medical rota, whereas others had a role based within their own sphere of professional practice (e.g. nurse consultants). Student participants ranged from those undertaking their first module of the MSc programme to those in their final year. In addition, some were studying modules on a free-standing basis.

Three key themes were identified: Recognising advanced practice; Education for Advanced Practice; Programme delivery (see Table 2). 
Table 2. Themes, categories and codes

\begin{tabular}{llcl}
\hline Themes & Recognising Advanced practice & Education for Advanced Practice & Programme delivery \\
\hline Categories & $\bullet$ Labels & $\bullet$ Flexibility & $\bullet$ Structure \& content \\
\& Codes & - Specialist v.s. advanced & - Overlap & - Core v.s. option modules \\
& $\bullet$ Requirements & $\bullet$ Rationale & - Pre-requisites \\
& $\bullet$ Role influence & - Constraints \& pressures & - Role relevance \\
& - Austerity & - Timing \\
& - Balancing education \& & - Teaching \& assessment methodology \\
& practice & - Expertise \\
& - Mentorship \& support & - Face to face vs online \\
& & - Positives \\
& & - Simulated \& actual practice \\
& & - Wider focus \\
& & - Diversity \\
& & - Profession or speciality focus \\
& & - Shared resources \\
\hline
\end{tabular}

\subsection{Recognising advanced practice}

There was much debate about how to define an ACP, with discussions reflecting diversity in titles and how the role is implemented in clinical practice: "There are about 200 people with CNS or Nurse Practitioner and various types of titles throughout the Trust... It doesn't then surprise me if people are then struggling, perhaps, about what this is because we don't know whether they're a Nurse Practitioner, or Nurse Specialist, or whatever else" (Nurse, FG 5). The role was, however, better defined in some areas of practice: "I think, certainly within ED, it's been very well defined; where perhaps it hasn't in other areas" (Paramedic, FG 5). A lack of clarity between specialist and AP roles across and within professional groups was also evident.

Participants expressed a need for a generic approach to AP across disciplines, whilst allowing people to retain their own professional identity. Blurred professional boundaries and a 'hybrid' role were described: "I'm glad that I' $m$ in this hybrid role, really, because I want to develop my skills like a doctor... actually like an advanced practitioner but obviously I will take my allied health professional brain with me and will be an empathetic, kind, caring, normal practitioner" (Nurse, FG 4). Autonomy was also considered a key component: "It's something about the autonomy within the role, I think... I think the ANPs work much more by themselves" (Nurse, FG 5). In addition, ACPs were seen to have a responsibility to continually advance their knowledge and to educate junior colleagues.

There was agreement that many ACPs were working without additional pay or a recognised qualification (advanced practitioner is not a protected title in the UK); resulting in a lack of standardisation and role confusion. Participants were keen to have the role acknowledged through professional registration: "I won't be registered at the NMC [Nursing \& Midwifery Council-UK regulatory body] as an advanced practitioner...So you do it but you don't actually get acknowledgement of it" (Nurse, FG 4). Implications relating to legal and professional cover were also raised: "I guess you're in such a responsible role that if you do get pulled up in a court of law you can back yourself up that you've got the education" (Nurse, FG 4).

Many participants believed that ACP roles had developed as a result of the change in junior doctor hours and austerity measures:

Nurse 1: "A lot of it is the burden away from the medics... I know it sounds very cynical, but I do think some of it is the whole work burden."

Nurse 2: “Oh, absolutely. That's where a lot of it stemmed from" (FG 4).

This was, however, considered by some to have provided an opportunity for nurses to widen their scope of practice and to prove themselves: "I think it's another way of replacing medical posts in a time where it's difficult to recruit. It's great for nurses to upskill and take on some of the roles that, traditionally, we wouldn't have done. I think that's been absolutely fantastic for nursing...” (Nurse, FG 5).

\subsection{Education for Advanced practice}

There were mixed views regarding the pre-requisites for an ACP role. Some felt it important to have first completed Masters level education: "If we are practising in an advanced role, we should be actually having a Master's in advanced practice" (Nurse, FG 4). 
Others were more ambivalent stating that employers: "say, 'oh, it will be nice if you're working towards a Master's', but do you have to have one? No. You can be working towards it for the next 20 years if you want..." (Paramedic, FG 4). Some also believed that one could learn on the job:

Nurse 1: "I agree with you that the Advanced Practitioner should be given training before they actually take up the role and that actually gives a lot of confidence and strength for them too..."

Nurse 2: “...I agree with what you said but... you can develop on the job as well. I think, for me, it's individualised to some extent..." (FG 2)

There was, however, a general consensus that adequate prior clinical experience and knowledge within the speciality was vital: "You have a bit more breadth as well behind you and about how to manage your own caseload, your timing and all of those things... that doesn't come with just doing the academic side of it” (Nurse, FG 5).

For some, education was important to meet national, professional or employer requirements. Others described education as giving them the confidence to do the job: "I must say that that has greatly supported me when I took up the role as a Senior Nurse because I had the confidence that I was able to pick up any oncology or oncological conditions which needed immediate attention. Without that, I wouldn't have been able to" (Nurse, FG 2). It also helped them prove their worth to others (particularly doctors): "the capacity to surprise their medical colleagues with the depth and knowledge and understanding and if that is a product of the course that that happens more frequently then that's a great thing." (AHP, FG 3).

Depending on their profession, local policies and role, participants described receiving a range of different education and training, either as part of a Master's programme or via other routes. The need for flexibility in provision and acknowledgement of previous learning and education was strongly emphasised: "There's a lot of crossover and I think that's where, for me, after doing them back to back... by the end of it I will be quite over my credit points" (Nurse, FG 4).

This overlap was particularly evident in competency assessment requirements for specialist and AP posts and the challenges of joining up different educational and practice requirements were discussed at length: "It's just trying to link all of that stuff together and just quite how you do that...I don't want somebody to be signed off on their Respiratory assessment, only to have to do exactly the same thing two years later for another competency booklet... How many times do you need to be assessed as competent for the same thing?” (Nurse, FG 5).

Participants described the potential benefits associated with people designing pathways that suited their individual requirements: "People know what they want to learn and it's for them really to go and find that education and for you to perhaps just provide the technology or the tool" (Nurse, FG 3). The benefits of multidisciplinary education were also highlighted, including the need for academics to apply learning to different disciplines: "just an acknowledgement that it's not just nurses there... it would just be nice to have some acknowledgement" (Midwife, FG 2).

The importance of balancing education with clinical practice experience was also discussed: "The balance should be proper preparation for the Advanced Practitioner course; plus the clinical practical required for the skills and it has to match up... There has to be a theoretical, academic background within the pathway itself and there has to be a certain percentage of clinical practice" (Nurse, FG 2). Participants noted, however, the difficulty of finding suitable practice mentors and assessors: "... I think it is going to be quite difficult who is going to be your assessor [mmm] in that sense. Is it going to be your work colleague, is it going to be your manager who might not have much knowledge about the speciality? [Mm-hmm]. Is it going to be a medical consultant who again comes from a different angle?" (Nurse, Int1). There was some discussion about protected placement time: "Students could go to the clinical areas to have specific days where they go and practise. I think that would be good" (Nurse, FG 2). There was, however, also concern about getting the time and support to do this. How to maintain AP skills once they had been acquired was another issue: "you want to bring that back and tie it with the new skills that you've learnt but then you think, 'Actually, I need to do that in my own time,"' (Nurse, FG 4).

Financial pressures were considered a significant challenge: "the problem is that the NHS is so under-resourced that makes it very difficult" (Nurse, FG 4) and one participant stated: "I think everybody is nervous, particularly about Masters Programmes and the lack of money and we don't know what's going to happen from year to year" (Nurse, FG 5).

\subsection{Programme delivery}

Programme prerequisites, such as a basic knowledge of physiology and statistics, were suggested to ensure everyone starts with a similar baseline. Most participants also felt that applicants should have first completed a speciality module: "I think you'd want somebody who'd done a specialist course, as a sort of entry qualification" (Nurse, FG 5).

ISSN 1925-4040 E-ISSN 1925-4059 
Core elements of an AP programme were considered to be applied pathophysiology, advanced assessment, a combination of research/evidence based practice and a clinical portfolio. Quality, safety and leadership were also seen as important, with a number of participants highlighting that ACPs should be at the forefront of improving services: "Yeah, just see how you can actually move the service on... I think that's all part of being an Advanced Practitioner" (Midwife, FG 2).

Pathophysiology was considered important by most: “...I think that's applicable to everyone's practice. It's a universal skill that we should all know regardless of what sort of practitioner you are. I think we should all know the A\&P or the pathway behind everything. So I think they should be core." (Paramedic, FG 4) There was also consensus that pathophysiology and advanced assessment were needed early in a programme as precursors to many other elements e.g. prescribing. One participant stated: "tying those two together, advanced assessment and physiology, would be quite beneficial. . . because you are, I guess, technically seeing, diagnosing and assessing people so it's good to have those linked a bit." (Midwife, FG 4).

For adult nurses, the current programme was reported to offer optional modules that gave students 'freedom' to focus on their interests, but this was not the case for those from other professional groups. The issue of ending up with too many credits was also discussed, but seen as potentially necessary to fulfil both programme and professional practice requirements.

In terms of teaching methods, a shift away from face-to-face learning was met with scepticism. The opportunity to share, exchange and discuss ideas and experiences, afforded by attending university was seen as important. One participant stated: "I like coming to the uni... You learn from each other, not just from the lecturer..." (Nurse, Int1). Another said: "To be able to ask for clarification I think is quite valuable, even though it's annoying having to come in on a day off [laughter]" (Midwife, FG 2). E-learning was noted to have an important role in providing baseline information, either before starting a programme or prior to a session, but the responsibility of the student to engage with available resources was also highlighted: “if I didn't do the e-learning and I came to the class... I would have expected to not be then supplemented with all the information because it's duplication in a way" (Pharmacist, FG 2).

There was a strong call for more 'hands on' experience: "Practical focus as well and kind of studying it in college... then go back again and to kind of deepen the knowledge of what you've gained so I kind of liked that aspect" (Nurse, Int1). The value of simulated practice is also illus- trated by the following excerpt:

Nurse 1: "So I've used simulation... it wasn't the same as real life but the students who had never experienced... they said that it was a really good tool, they say it's a safe environment to get things but to know when to administer adrenalin..."

AHP 1: "I think it also has a role... so you can play with the tools, so you can understand, a more intuitive understanding of the data stream that's coming to you..." (FG 3)

More opportunities to examine real patients either in the classroom or in placement was requested: "Is there not a way to...recruit patients with certain pathophysiology to come in for the afternoon for a few hours... like two or three patients with murmurs to come in and then the students actually listen for the murmur..." (Pharmacist, FG 2). In addition, the use of experts both in the classroom and in practice was viewed as important: "Yeah, so [experts] their doing policy, leadership and delivery from NHS England and things" (Midwife, FG 2). Participants also pointed out the value of Faculty staff who are still in practice: "They still, you know, bring their practical hat with them [mmm] as well and I think that's quite important [mm-hmm] as well so having this link in a way to what it is, the workplace..." (Nurse, Int 1)

In terms of assessment, participants expressed a desire for a wider range of methods that addressed all elements of the ACP role. Objective Structured Examination Stations (OSCES) were seen as particularly valuable:

Nurse 1: "But I do think it needs to be definitely OSCE based."

Paramedic 1: "Not so much assignments... why can't you assess us with another method? An exam, an OSCE?"

Nurse 1: “... Because at the end of the day you are going to be out seeing people and examining people so it's nice to put that into perspective in the training and the teaching; get people to demonstrate how they do it in a real clinical environment." (FG 4)

Other practice focused assessment methods were also perceived as useful: "Instead of an essay... an audit against the national standard which is quite useful because then that's something you can take back to work on and you could reaudit against; so things that are academic but also practical" (Nurse, FG 5). 
There was consensus that future programmes needed to focus on recruiting and meeting the needs of a multi-disciplinary student cohort. One participant said: "I think it's really nice to have different healthcare professionals... And, you know, it kind of just opens your horizon a little bit more" (Nurse, Int1). Another agreed saying: "I think that going forward to the future I think the programmes need to be multidisciplinary” (Nurse, FG 3). Having an AP programme located in a Faculty representing only one professional group was, however, considered detrimental to the recruitment of a wider cohort: “... So I think that this school of nursing and midwifery... I think it's all geared towards nursing because it's a nursing school predominantly... ” (Paramedic, FG 4). Cross faculty provision was also viewed as an important way to promote collaboration and enable sharing of resources: “... I mean there's some overlapping and some sharing but there isn't enough joint education going on and I think on an equal basis." (Nurse, FG 3)

\section{Discussion}

The aim of this qualitative study was to explore the training and education needs of ACPs. Three key themes were identified: recognising AP; education for AP and programme delivery.

\subsection{Implications for education, practice and policy}

- Future programmes should be designed based on national and/or international frameworks and should focus on the needs of all professional groups and specialities

- Flexible, blended approaches to teaching, learning and assessment, with a focus on clinical practice priorities are recommended to meet the individual requirements of a diverse student body

- Collaborative, partnership working is essential for the development of future ACPs

- Clinical practice areas need to understand and support the requirements for role modelling, mentorship and assessment

- The pros and cons of statutory regulation for ACPs and its implications for future education need to be critically considered by policy makers.

To shape any curriculum, it is necessary to define the population of interest. In line with other international authors, ${ }^{[12,13]}$ we identified a myriad of ACP labels and titles. Within the nursing profession in the UK, for example, there is currently no statutory regulation by the Nursing and Midwifery Council, hence anyone can call themselves an advanced practitioner. $^{[14,15]}$ To address this, HEIs need to design curricula based on national and/or international frameworks. ${ }^{[1,2]}$
Health Education England (HEE) identify four key pillars of AP: clinical practice; management and leadership; education and research; and clinical competence. ${ }^{[2]}$ Support for these elements was reflected in the data we collected. Our findings suggest that core modules should include research, advanced assessment skills, applied pathophysiology and an understanding of quality, safety and leadership, with additional optional modules, which enable individuals to focus on their specific practice needs.

Our findings support the benefits of a more diverse cohort to both the individual and to the promotion of effective inter-professional work. Although ACPs need to be able to work autonomously, inter-professional education and practice needs to be fostered and encouraged to improve patient safety. ${ }^{[16,17]}$ To attract a more diverse student cohort, programmes must meet the needs of all professional groups and specialities. The current system was seen to provide good choice for adult nurses but wanting when applied to other professional groups. Student satisfaction is a key outcome marker of quality for HEIs; attention to this is vital to secure funding, to attract future students and to maintain a good reputation. ${ }^{[18]}$

To support ACPs' management of a complex clinical caseload, partnership working is required. ${ }^{[2]}$ Improved collaboration across faculties within HEIs and with external organisations such as NHS partners would facilitate the provision of content applicable to all professional groups; increase student opportunities; promote sharing of expertise and; potentially reduce the financial burden. Data from a focus group study $(n=67)$ by Jones et al. support this view, with authors highlighting the need for more 'joined-up' thinking, support and development opportunities. ${ }^{[5]}$ There are, however, significant challenges to cross boundary working, with success frequently hampered by restrictive structures and processes. These problems have been described in literature related to achieving integrated care within the $\mathrm{NHS}^{[19]}$ and across other large-scale organisations.

In a system struggling to fund continued professional development, where students find it hard to balance the demands of work and study, our findings and those of others suggest that greater flexibility and shared working is key to producing future ACPs who are fit for purpose. ${ }^{[5,16]}$ New models of ACP education, such as the apprenticeship standard currently in development in England ${ }^{[20]}$ also need to be critically reviewed to determine their benefits and the degree to which there is 'organisational readiness' for work based approaches. ${ }^{[21]}$ In addition, consideration must be given to any stipulations with regard to potential future registration of some titles by different professional bodies (for example, the 
consultation on medical associate roles by the Department of Health \& Social Care). ${ }^{[22]}$

Our findings support the use of a blended approach to teaching and learning. Contact time with both academics and practitioners is essential to develop and test ideas and the use of models such as clinical internships are suggested. ${ }^{[23]}$ E-learning is a valuable way to help prepare for and supplement learning but is not an effective substitute for time spent with experts; be they patients, lecturers or practice staff.

Using practice focused assessment methods and avoiding unnecessary overlap were identified as key issues. Health care professionals frequently have to complete a number of different competency documents during their career (for example, the integrated competence framework published by the Royal College of Nursing), many of which have the same or similar elements. ${ }^{[24]}$ This creates significant workload for both the individual and assessors. Students should be provided with the resources needed to map previous competencies. ${ }^{[25]}$ National recommendations describing who can assess clinical competence are also required. As also identified highlighted by Bergström and Lindh, a further issue relates to expanding the pool of appropriate role models and determining what previous qualifications and experience are required to perform a mentorship role. ${ }^{[7]}$

\subsection{Limitations}

This small scale qualitative study is limited by a number of factors. It sought only the views and experiences of students studying AP at one HEI or ACPs working in local NHS hos- pitals within England. Furthermore, despite several attempts to improve recruitment, the final sample included only 17 people, most of whom were nurses. The myriad of different roles and titles also made it challenging to achieve consensus. Together with a review of the current literature, we believe, however, that our data provide a rich and insightful picture of the current local picture, which will be of value to other HEIs and their clinical partners, both within and beyond the UK.

\section{Conclusion}

This qualitative study explored views and experiences related to the education and training needs of ACPs. Findings highlight the huge variation in titles, practice responsibilities and management structure, which make the development of a generic programme challenging and the importance of flexibility key to success. At a time when public services are experiencing significant financial constraints, the need for improved collaboration, shared resources and a practice focus is vital to educating ACPs who will be fit for the future.

\section{ACKNOWLEDGeMENTS}

This work was funded by the Florence Nightingale Faculty of Nursing, Midwifery \& Palliative Care, King's College, London.

\section{Conflicts of InTEREST Disclosure}

The project was funded by the Higher Education Institution, which hosts the AP programme and the project team included academics from the Faculty.

\section{REFERENCES}

[1] International Council of Nurses (ICN). Definition and Characteristics of the Role. Nurse Practitioner/Advanced Practice Network. 2015. Available from: https://international.aanp.org/Practic e/APNRoles

[2] Health Education England (HEE). Multi-professional framework for Advanced Clinical Practice in England. 2017. Available from: https://www.hee.nhs.uk/our-work/advanced-clini cal-practice/multi-professional-framework

[3] Department of Health (DH). Advanced level nursing: a position statement. 2010. Available from: https://www.gov.uk/government/uploads/system/u ploads/attachment_data/file/215935/dh_121738.pdf

[4] Hall J, Katz S, Cor M. Patient satisfaction with pharmacist-led collaborative follow-up care in an ambulatory rheumatology clinic. Musculoskeletal Care. 2016. https://doi .org/10.1002/msc. 1160

[5] Jones A, Powell T, Watkins D, et al. Realising their potential? Exploring interprofessional perceptions and potential of the advanced practitioner role: a qualitative analysis. BMJ Open. 2015; 5(12). https://doi.org/10.1136/bmjopen-2015-009740

[6] Imison C, Castle-Clarke S, Watson R. Reshaping the workforce to deliver the care patients need. Nuffield Trust: London. 2016.

[7] Bergström P, Lindh V. Developing the role of Swedish advanced practice nurse (APN) through a blended learning master's program: Consequences of knowledge organisation. Nurse Education in Practice. 2018; 28: 196-201. https://doi.org/10.1016/j.nepr.2 017.10 .030

[8] Fagerström L. Developing the scope of practice and education for advanced practice nurses in Finland. International Nursing Review. 2009; 56(2): 269-272. https://doi.org/10.1111/j.1466-765 $7.2008 .00673 . \mathrm{x}$

[9] Jamieson L, Williams L. M. Focus group methodology: explanatory notes for the novice nurse researcher. Contemporary Nurse. 2003; 14(3): 271-280. PMid:12868666 https://doi .org/10.5172/co $\mathrm{nu} .14 .3 .271$

[10] Litosseliti L. Using focus groups in research. London: Continuum; 2007.

[11] Newell R, Burnard P. Research for evidence based practice in healthcare. 2nd edition. London: Wiley Blackwell; 2011.

Published by Sciedu Press 
[12] Heale C, Rieck Buckley C. An international perspective of advanced practice nursing regulation. International Nursing Review. 2015; 62(3): 421-429. https://doi.org/10.1111/inr.12193

[13] Parker J, Hill M. A review of advanced practice nursing in the United States, Canada, Australia and Hong Kong Special Administrative Region (SAR), China. International Journal of Nursing Sciences. 2017; 4(2): 196-204. https ://doi.org/10.1016/j.ijnss. 2017.01 002

[14] Oliver E. Advanced practitioners improve efficiency and patient satisfaction. Independent Nurse. 2017.

[15] Rolfe G. Advanced nursing practice 1: understanding advanced nursing practice. Nursing Times. 2014; 110 (27): 20-23.

[16] Lo L. Teamwork and Communication in Healthcare a Literature Review. Canadian Patient Safety Institute. 2011. Available from http://www.patientsafetyinstitute.ca/en/toolsResour ces/teamworkCommunication/Documents/Canadian\%20Fra mework $\% 20$ for $\% 20$ Teamwork $\% 20$ and $\% 20$ Communications $\% 20 \mathrm{~L}$ it\%20Review.pdf

[17] Raleigh M, Allan H. A qualitative study of advanced nurse practitioners' use of physical assessment skills in the community: shifting skills across professional boundaries. Journal of Clinical Nursing. 2016; 26(13-14): 2025-2035. https ://doi.org/10.1111/jocn .13613

[18] Higher Education Funding Council for England (HEFCE). National Student Survey. 2017. Available from: http://www.hefce.ac.u $\mathrm{k} / \mathrm{lt} / \mathrm{nss} / \mathrm{results} /$
[19] West M. Conflict and collectivism: the challenges of working across boundaries in health care. 2015. Available from: https : //www.kingsfund.org.uk/blog/2015/03/conflict -and-collectivism-challenges-working-across-bound aries-health-care

[20] Skills for Health. Advanced Clinical Practitioner Apprenticeship standard. 2018. Available from: https://haso.skillsforhealth.o rg.uk

[21] Livesley J, Waters K, Tarbuck P. The management of advanced practitioner preparation: a work-based challenge. Journal of Nursing Management. 2009; 17(5): 584-93. https://doi.org/10.1111/ j.1365-2834.2009.00967.x

[22] Department of Health and Social Care. The regulation of medical associate professions in the UK. 2017. Available from: https://www.gov.uk/government/consultations/re gulating-medical-associate-professions-in-the-uk

[23] Lee G, Fitzgerald L. A clinical internship model for the nurse practitioner programme. Nursing Education in Practice. 2008; 8: 397-404 https://doi.org/10.1016/j.nepr.2008.03.002

[24] Royal College of Nursing. Integrated core career and competence framework for registered nurses. 2009. Available from: https://www.rcn.org.uk/professional-developme $\mathrm{nt} / \mathrm{publications/pub-003053}$

[25] O'Connell J, Gardner G, Cryer F. Beyond competencies: using a capability framework in developing practice standards for advanced practice nursing. Journal of Advanced Nursing. 2014; 70(12): 27282735. https://doi.org/10.1111/jan.12475 